\title{
Purificación Parcial y Caracterización de Alfa Amilasa de granos germinados de Chenopodium quinoa (Quinua)
}

\section{Partial Purification and Characterization of Alpha Amylase from germinated grains from Chenopopdium quinoa (Quinua)}

\author{
Melissa Bedón Gómez, Oscar Nolasco Cárdenas, Carlos Santa Cruz C. y Ana I. F. Gutiérrez Román \\ Universidad Nacional Federico Villarreal, Facultad de Ciencias Naturales y Matemática, Laboratorio de \\ Bioquímica y Biología Molecular, Jr. Río Chepén S/N, El Agustino. Telefax: 362 - 3388
}

DOI: https://doi.org/10.33017/RevECIPeru2013.0007/

\section{Resumen}

Las alfa amilasas son las enzimas más estudiadas e importantes en el campo biotecnológico e industrial; ya que han reemplazado por completo la hidrólisis química del almidón. Estas enzimas son imprescindibles en la elaboración de productos alimenticios, combustibles, medicamentos y detergentes con la finalidad de optimizar procesos y conservar el medio ambiente. La $\alpha$-amilasa puede ser purificada de diferentes organismos como plantas, animales, hongos y bacterias; actualmente un gran número de $\alpha$-amilasas bacterianas en especial del género Bacillus están disponibles comercialmente y son las más utilizadas en las industrias. Sin embargo, la producción de éstas no satisfacen los requerimientos industriales en el mundo; ya que, la demanda de esta enzima se ha incrementado en los últimos dos años y el empleo de $\alpha$-amilasas bacterianas ha provocado alergias afectando al $15 \%$ de la población a nivel mundial. . En este estudio, como fuente de $\alpha$-amilasa se emplearon semillas de Chenopodium quinoa (quinua) var hualhuas blanca durante el proceso de germinación; esta enzima fue parcialmente purificada por precipitación con sulfato de amonio obteniendo una actividad específica final de $35.60 \mathrm{U} / \mathrm{mg}$ y un grado de purificación de 5 veces. La purificación fue confirmada por SDS-PAGE, encontrando un peso molecular de 44kDa. La actividad enzimática se evaluó mediante el método de Miller mostrando máxima actividad a $\mathrm{pH} 7$ y a temperatura de $37^{\circ} \mathrm{C}$. La linealización de Lineweaver-Burk nos dio un $\mathrm{Km}$ de $16 \mathrm{mg} / \mathrm{mL}$ y Vmax de $100 \mu \mathrm{M}$ de maltosa/min. Por lo tanto, esta caracterización reúne los pre-requisitos necesarios para la aplicación en la industria.

Descriptores: Chenopodium quinoa; alfa amilasa; germinación; purificación parcial.

\begin{abstract}
The alpha amylases are the enzymes most studied and important in biotechnology and industry; because they have completely replaced the starch's chemical hydrolysis. These enzymes are essential in the food production, medicines and detergents in order to optimize processes and conserve the environment. The $\alpha$ amylase can be isolated from different organisms such as plants, animals, fungi and bacteria, now a large number of bacterial $\alpha$-amylases especially from genus Bacillus are commercially available and they are the most used in industry. However, the production of these do not meet industry requirements in the world, because the demand for this enzyme has increased in the last two years and the use of bacterial $\alpha$-amilase has caused allergies affecting the $15 \%$ of the global population. In this study, as a source of a-amylase used the seeds from Chenopodium quinoa (quinoa). Var. white hualhuas during the germination process, this enzyme was partially purified by ammonium sulfate precipitation to obtain a final specific activity of $35.60 \mathrm{U} / \mathrm{mg}$, and a grade of purification of 5 times. The purification was confirmed by SDS-PAGE, where the molecular weight was $44 \mathrm{kDa}$. The enzyme activity was evaluated by Miller method showing maximum activity at $\mathrm{pH} 7$ and $37^{\circ} \mathrm{C}$. The Lineweaver-Burk linearization shows a $\mathrm{Km}$ of $16 \mathrm{mg} / \mathrm{mL}$ and Vmax of $100 \mu \mathrm{M}$ the maltose / min. Therefore, these characterizations meet the prerequisites need for industry.
\end{abstract}


Keywords: Chenopodium quinoa; alpha amylase; germination; partial purification

\section{Introducción}

La quinua (Chenopodium quinoa) es un pseudocereal nativo de América del sur $y$ actualmente el Perú es considerado uno de los países con mayor producción; especialmente en los departamentos de Puno, Cuzco, Ayacucho, Apurímac, Huancavelica y Junín. (MINAG-2012), y se presume que la tasa de producción para el año 2013 se incrementará el 15\% hasta llegar al 48,000 TN. Desde el punto de vista nutricional la quinua posee un excepcional equilibrio de proteínas, grasa, aceite y almidón, así como un alto grado de aminoácidos esenciales [1-3]. El almidón es el mayor componente de las semillas de quinua y comprende aproximadamente el $55 \%$ de su peso (Lindeboom, 2005). Este carbohidrato es la principal fuente de energía e importante para el desarrollo y crecimiento de la planta; ya que, cuando las semillas inician el proceso de germinación, el almidón comienza a degradarse por acción de las a amilasas; con la finalidad de proporcionar las moléculas de glucosa que serán oxidadas para producir la energía necesaria para el desarrollo del embrión. (Conn et al., 2001).

Las $\alpha$-amilasas son las enzimas más estudiadas e importantes en el campo biotecnológico e industrial; ya que han reemplazado por completo la hidrólisis química del almidón (Polisacárido más explotado a nivel mundial) (Monteiro de Souza \& De Oliveira, 2010). Esta enzima es imprescindible en la elaboración de productos alimenticios, combustibles, medicamentos y detergentes con la finalidad de optimizar procesos y conservar el medio ambiente. En la industria alimentaria, la $\alpha$-amilasa es ampliamente empleada en la elaboración de pasteles, preparación de digestivos, jarabes, cerveza y panadería (Guadarrama et al., 2009); esta industria invierte anualmente millones de euros en la elaboración de productos libres de sustancias químicas altamente contaminantes, ya que esto podría ocasionar problemas de eutrofización, ecotoxicidad y efectos sobre la salud humana. (Martínez, 2005).

La a-amilasa puede ser purificada de diferentes organismos como plantas, animales, hongos $y$ bacterias; actualmente un gran número de aamilasas bacterianas están disponibles comercialmente y son las más utilizadas en las industrias, debido a que son considerados más estables a altas temperaturas. (Monteiro de Souza \& De Oliveira., 2010). Sin embargo, las producciones de estas enzimas no satisfacen los requerimientos industriales y de salud en el mundo; Además, la utilización de $\alpha$-amilasas bacterianas en la industria alimentaria ha provocado sensibilidad (alergias) en la población, como es el caso del "asma del panadero". Por lo tanto, la creciente demanda de esta enzima en el campo industrial ha establecido un hecho que evidencia la necesidad de buscar nuevas alternativas de $\alpha$ - amilasa con una eficiente actividad y que no provoquen efectos negativos sobre la salud humana.

En el presente estudio, el objetivo principal fue purificar parcialmente y caracterizar la alfa amilasa de granos germinados de Chenopodium quinoa (quinua) var. hualhuas blanca, con la finalidad de demostrar que la a-amilasa de quinua presenta características cinéticas similares a los cereales. Para cumplir con dicho objetivo, se determinó el tiempo de germinación de las semillas donde la actividad de la a-amilasa es mayor, el peso molecular estimado, la afinidad de la $\alpha$-amilasa por el almidón soluble y la temperatura y el $\mathrm{pH}$ óptimo de la $\alpha$ amilasa.

\section{Materiales y Métodos}

\section{Muestra Biológica}

Se emplearon semillas de Chenopodium quinoa (quinua) var. hualhuas blanca provenientes de la ciudad de Huancayo-departamento de Junín, que fueron seleccionados según el tamaño del grano (mayores a 1,6 $\mathrm{mm}$ de diámetro), se desinfectaron con hipoclorito de sodio $(\mathrm{NaOCl})$ al $1 \%$ durante 5 minutos y posteriormente fueron lavados hasta retirarlo completamente, quedando estos listos para su germinación.

\section{Determinación del tiempo de germinación}

Para determinar el tiempo de germinación donde la actividad de la $\alpha$-amilasa de quinua es mayor; se utilizaron placas petri con solución de estreptomicina y gentamicina, $(5 \mu \mathrm{g} / \mu \mathrm{l}$ cada uno). El tiempo de incubación de cada muestra fue variable $(0,3,6,9$, $12,15,18,24,30,36,48$ y 72 horas) y se realizó en una cámara oscura, a una temperatura de $22^{\circ} \mathrm{C}$ y $83 \%$ de humedad relativa. Las semillas previamente germinadas fueron recolectadas y homogenizadas (extracto crudo), en cada extracto se determinó la cantidad de proteínas totales y la actividad enzimática de la a-amilasa. Por otro lado, se evaluó el porcentaje de germinación y almidón a cada tiempo de germinación. Para ello se utilizó una 
cámara de germinación donde se puede variar la temperatura y mantener la humedad relativa constante.

\section{Preparación del extracto crudo}

El método que se tuvo en cuenta para la preparación del extracto crudo fue según Machaiah (Machaiah \& Vakil, 1984), en la cual, las semillas previamente germinadas fueron trituradas con Buffer fosfato $20 \mathrm{mM}$ con $6.7 \mathrm{mM}$ de cloruro de sodio a $\mathrm{pH}, 6.9$ atemperado a $4^{\circ} \mathrm{C}$ en relación de $(1 \mathrm{w}: 5 \mathrm{~V})$. El filtrado obtenido se centrifugó a $2000 \mathrm{~g}$ durante 20 minutos, descartando el precipitado. El sobrenadante se centrifugó nuevamente a $4500 \mathrm{~g}$ durante 5 minutos recuperando el sobrenadante. Se utilizó centrifuga refrigerada a $4^{\circ} \mathrm{C}$.

\section{Extracción y purificación parcial de la enzima}

Previamente determinado el tiempo de germinación, se colocaron a germinar semillas en las mismas condiciones y el extracto crudo $u$ homogenizado con buffer fosfato (1w:3v) fueron ajustados a $45 \%$ de saturación con sulfato de amonio (NH4)2SO4. La precipitación proteica se realizó durante 15 horas a $4^{\circ} \mathrm{C}$. Posteriormente el precipitado y el sobrenadante fueron desalinizados y concentrados mediante tubos con filtros Amicon Ultra 10K-NMWL de 10000 . El sobrenadante obtenido se dejó precipitando con sulfato de amonio a $60 \%$ de saturación durante 15 horas a $4^{\circ} \mathrm{C}$. La separación del precipitado, la desalinización y la concentración se realizó siguiendo el mismo procedimiento ya descritos anteriormente.

\section{Electroforesis en gel de poliacrilamida SDS-PAGE}

El protocolo de electroforesis utilizado fue el establecido por Laemmli en 1970 (Ausubel \& Brent, 2003). La electroforesis se realizó con la finalidad de determinar el peso molecular estimado de la $\alpha$ amilasa de Chenopodium quinoa (quinua).El sistema de electroforesis empleado fue de geles de dos fases, la fase de apilación o de stacking fue de $4.5 \%$ de gel poliacrilamida y la fase de resolución al $12 \%$ de poliacrilamida. Se utilizó un equipo de electroforesis vertical.

\section{Cuantificación de proteínas totales}

En cada una de las muestras y fracciones obtenidas durante el proceso de purificación de a-amilasa, se determinó la concentración de proteínas utilizando el método de Gornall et al., 1947 y un espectrofotómetro Shimatzu 1700.

Evaluación de actividad enzimática
Los germinados fueron cosechados para la extracción de enzima que usa $0.02 \mathrm{M} \mathrm{NaH}_{2} \mathrm{PO}_{4}$, en $\mathrm{pH} 6 \cdot 9$, con $0.006 \mathrm{~m} \mathrm{NaCl}$ y el extracto fue usado en el ensayo. El método utilizado para evaluar la actividad enzimática de la $\alpha$-amilasa fue el método de Miller (DNS), cuyo protocolo fue el establecido por sigma - Aldrich, donde el almidón primero fue convertido a maltosa catalizado por la alfa-amilasa. Luego a la maltosa se le hace reaccionar con el ácido 3, 5-dinitrosalicilico, formando un producto coloreado con máxima absorción en 540 nm, cuando esta reducido. Los valores de la absorción fueron leídos sobre una curva estándar establecida con el aumento de las cantidades de maltosa La concentración de proteína total fue determinada por método de biuret (Gornall et al., 1949), y la actividad expresada en unidades por mg proteína. Una unidad de actividad de alfa-amilasa es definida como $\mu \mathrm{m}$ de maltosa producido por minuto y la actividad específica fue expresado en términos de unidades por mg de proteína.

\section{Caracterización Cinética de la $\alpha$-amilasa}

Para su respectiva caracterización se evaluó el efecto de temperatura y $\mathrm{pH}$ sobre la velocidad de reacción y los parámetros cinéticos de la amilasa fueron determinados a $\mathrm{pH} 7$ y a $37^{\circ} \mathrm{C}$. Los valores de la constante de Michaelis $(\mathrm{Km})$ y la velocidad máxima fueron determinados mediante la linealización de Lineweaver-Burk. Equipos utilizados: fueron un baño maría graduable de temperatura y un espectrofotómetro.

\section{Análisis estadístico}

Para determinar las diferencias significativas y las correlaciones entre los valores obtenidos o variables se utilizó la prueba no paramétrica de Kruskall wallis y la prueba de correlación de Tau Kendall mediante el programa estadístico SPSS (Statistical Package for the Social Sciences).

\section{Resultados y Discusión}

Determinación del tiempo de germinación vs. La Máxima Actividad enzimática.

En la figura 1 se muestra los valores actividad amilásica del extracto crudo de las semillas germinadas de Chenopodium quinua a diferentes tiempos (0-72 horas). Se puede apreciar que las semillas germinadas de 36 horas son las que presentan mayor valor de actividad específica $(24.345 \mathrm{U} / \mathrm{mg})$ y el menor valor fue obtenido a las 0 Horas de germinación (8.953U/mg).(Figura 1). 
$\left(P=0.025^{*}\right) . *$ : La actividad enzimática se incrementa significativamente a las 36 horas de germinación con 95\% de confiabilidad. De cada uno de los tiempos de germinación evaluados se colocaron $100 \mu \mathrm{g}$ de proteínas en cada posillo de un gel de SDS-PAGE al $12 \%$, en esta imagen se aprecia que la enzima $\alpha$ amilasa cuyo peso molecular estimado fue de $44 \mathrm{kDa}$ presenta una progresiva aparición en las primeras horas de germinación (0-36 horas) y un descenso en las últimas horas (42-72 horas). Nuestros resultados concuerdan con el estudio de otros autores en otros cereales, como arroz, soja, donde la actividad específica de la enzima aumentó a un máximo después de la inbibición y después disminuyó (Ismail et al., 2009; Kumari et al., 2010)

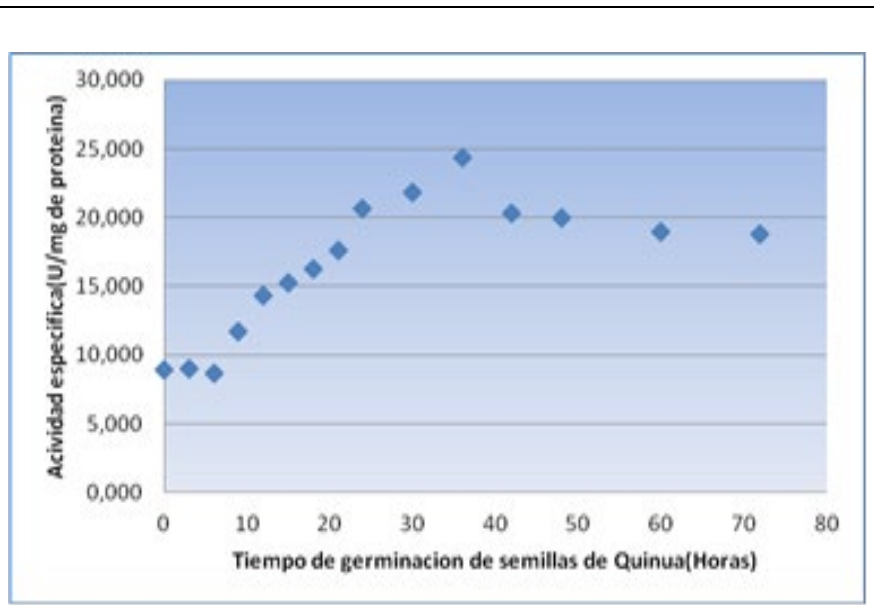

Figura 1: Actividad específica del extracto crudo de semillas de Chenoponium quinoa var. hualhuas blanca germinadas a diferentes horas.

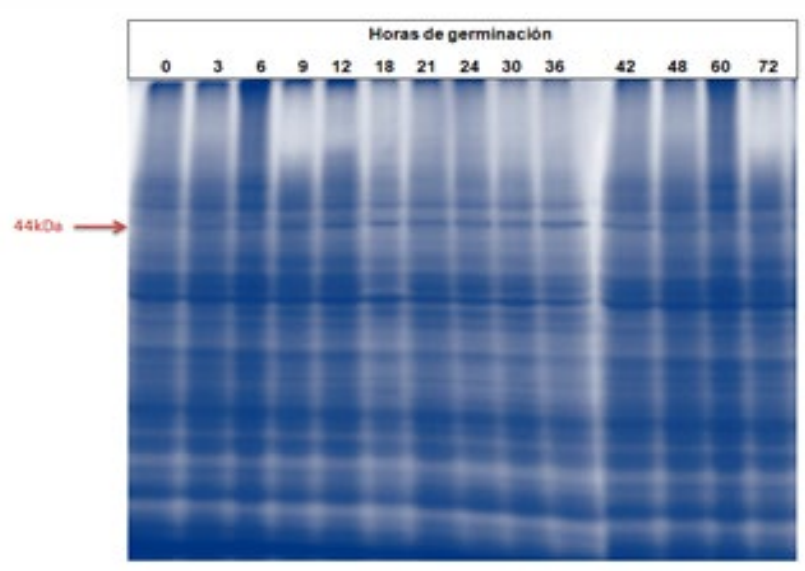

Figura 2: Corrida electroforética SDS-PAGE-12\% de los extractos crudos de las semillas de Chenopodium quinoa var. hualhuas blanca germinadas a diferentes horas.
Evaluación del grado de purificación y rendimiento (\%) de las fracciones obtenidas durante el proceso de purificación de la $\alpha$-amilasa

La tabla 1 muestra el rendimiento de proteínas y el grado de purificación de las fracciones obtenidas durante el proceso; en la cual, se determinó que la fracción precipitado 2 (P2) presenta mayor actividad específica $(35,60 \mathrm{U} / \mathrm{mg})$ con un grado de purificación de 5.0 veces y rendimiento de proteínas de $30 \%$. Además, la fracción de menor actividad específica es el sobrenadante 2 (S2) con $5.38 \mathrm{U} / \mathrm{mg}$. La actividad específica de la fracción P2 muestra una diferencia significativa respecto al extracto crudo. $(P<0.01)$ con el $99 \%$ de confiabilidad según la prueba de Kruskal Wallis. Nuestro resultado se asemeja a los resultados obtenidos por Machaiah y Espinel donde, su mejor rendimiento de purificación de la a-amilasa lo obtuvieron en el rango de 45 y $70 \%$ de saturación respectivamente. (Machaiah, 1984 \& Espinel, 2009), además, tenemos datos que indican que en la germinación de soja donde usaron el fraccionamiento con sulfato de amonio dio la purificación de 6.7 veces (Kumari et al., 2010), lo cual posiblemente ayudó a eliminar a los inhibidores de las amilasas alfa (Wisessing et al., 2010).

Tabla 1: Resumen de la purificación de a-amilasa de semillas germinadas de Chenopodium quinoa var. hualhuas blanca.

\begin{tabular}{|c|c|c|c|c|c|c|}
\hline Fracciones & $\begin{array}{c}\text { Volumen } \\
\text { total } \\
\text { (mL) }\end{array}$ & $\begin{array}{l}\text { Protcina } \\
\text { (mg) }\end{array}$ & $\begin{array}{c}\text { Actividad } \\
\text { cnzimátioa } \\
\text { U } \\
\text { (umolml) }\end{array}$ & $\begin{array}{c}\text { Rendimicnto } \\
(\%)\end{array}$ & $\begin{array}{c}\text { Actividad } \\
\text { especifica } \\
\text { (U/mg) }\end{array}$ & $\begin{array}{l}\text { Grado de } \\
\text { Purificación }\end{array}$ \\
\hline Extracto & & & & & & \\
\hline Crudo & 20 & 191,9 & 32,0 & 100 & 6,69 & 10 \\
\hline $\begin{array}{l}\text { Precipitado } 1 \\
( \pm 45 \%) \\
\text { Sobrenadante }\end{array}$ & 10 & 18,7 & 25,3 & 26.1 & 27,15 & 4,06 \\
\hline $\begin{array}{l}1\left(^{*} 45 \%\right) \\
\text { Sobrenadante }\end{array}$ & 20 & 68,8 & 23,2 & 70,4 & 13,50 & 2,02 \\
\hline $\begin{array}{l}2\left(^{\prime} 60 \%\right) \\
\text { Precip itado } 2\end{array}$ & 8 & 28,1 & 9,4 & 40,5 & 0,38 & 0,80 \\
\hline$(+60 \% \div)$ & 12 & 14,7 & 21,8 & 30.1 & 35,60 & 5,32 \\
\hline
\end{tabular}

${ }^{*}$ Porcentaje de saturación con sulfato de amonio

\section{Determinación del peso molecular de la $\alpha$-amilasa}

La comparación de la movilidad electroforética de la a-amilasa de Chenopodium quinoa var. hualhuas blanca con la de los marcadores de peso molecular conocidos permitió establecer un peso molecular de 44 kDa (Rf: 0.313) tal como se muestra en la figura 3 y 4 . Tomando en cuenta los estudios de Warner, esta enzima correspondería a una de las diferentes formas de amilasas que se encuentran en granos germinados de cereales y que sus pesos moleculares están alrededor de 42 a 45 kDa. (Warner, 1991). Además, la a-amilasa de quinua 
presenta un peso molecular similar a la de Oriza sativa (arroz) (40 kDa) obtenido por Ziegler (Ching Lee, 2001).y la de Triticum sp. (43 kDa) (Machaiah \& Vakil, 1984).

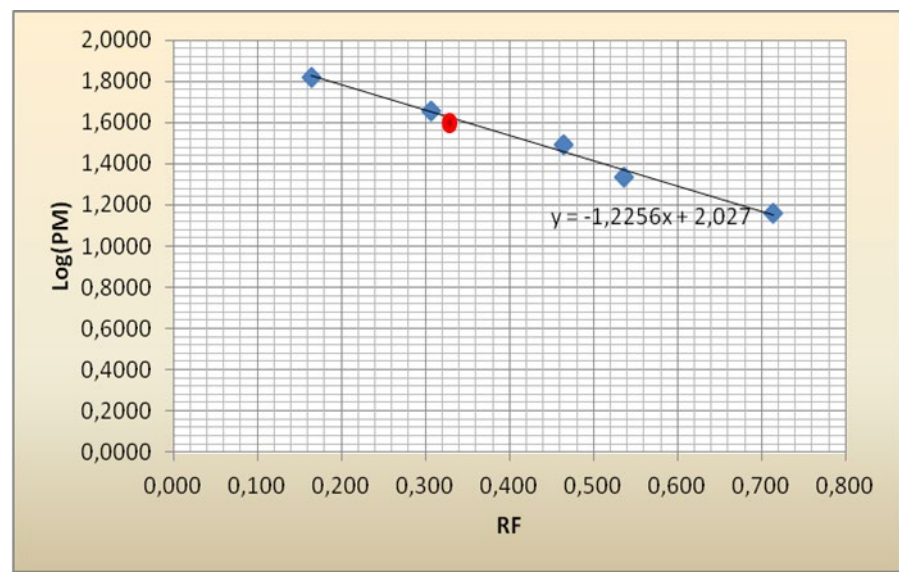

Figura 3: Representación gráfica de la extrapolación entre el Rf y el Logaritmo del peso molecular de proteínas estándar y el valor de RF de Chenopodium quinuoa var, hualhuas blanca.

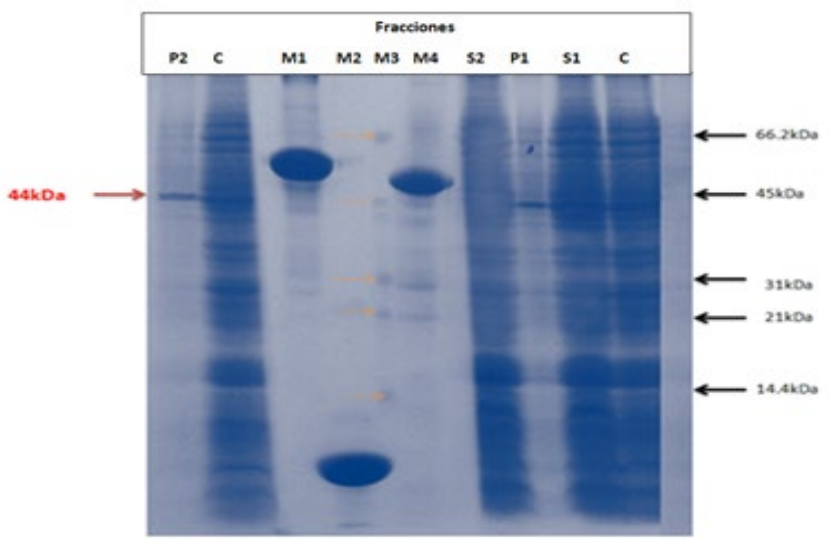

Figura 4: Corrida electroforética SDS-PAGE-12\% de las fracciones obtenidas durante el proceso de purificación de la enzima a-amilasa de Chenopodium quinoa var. hualhuas blanca. En Carril P2: Precipitado 2 (60\%), Carril C: extracto crudo, carril M1: BSA, carril M2: Lizosima, carril M3: marcador Biorad, carril M4: a-amilasa de Bacillus licheniformis. Carril S2: Sobrenadante 2, Carril P1: Precipitado 1 (45\%), Carril S1: Sobrenadante 1.

\section{Caracterización Cinética de la $\alpha$ - amilasa}

Efecto de la concentración de sustrato sobre la velocidad de reacción de la enzima Determinación de la velocidad máxima (Vmax) y $\mathrm{Km}$

Según la representación de Lineweaber-Burk (fig.5), se pudo determinar los valores de $\mathrm{Km}$ y Vmax. Donde $-1 / \mathrm{Km}=-0.06$ y $1 / \mathrm{Vmax}=0.01$. Por lo tanto: la velocidad máxima de la reacción es $100 \mu \mathrm{M} / \mathrm{min}$ y la concentración de sustrato donde la enzima funciona a la mitad de la velocidad máxima $(\mathrm{Km})$ es 16 $\mathrm{mg} / \mathrm{mL}$. La a-amilasa de Chenopodium quinoa var. hualhuas blanca posee $75 \%, 56.3 \%$ y $50 \%$ de afinidad por el almidón soluble, comparada con las $\alpha$ amilasas de granos como: Zea maiz (maíz) $(\mathrm{Km}=12.5$ $\mathrm{mg} / \mathrm{mL})$, de Panicum miliaceum (mijo) $(\mathrm{Km}=9 \mathrm{mg} / \mathrm{mL})$ y Triticum sp (trigo) $(\mathrm{Km}: 8 \mathrm{mg} / \mathrm{mL}$ ), (Adewale et al., 2011). Su afinidad es mucho menor si la comparamos frente a las $\alpha$-amilasas microbianas como Bacillus sp, Aspergillus fumigatus y Thermofibida pullulans cuyos valores de $\mathrm{Km}$ son relativamente bajos $(1.64 ; 0.42$ y $0.88 \mathrm{mg} / \mathrm{mL}$ respectivamente) (Espinel \& López, 2009).

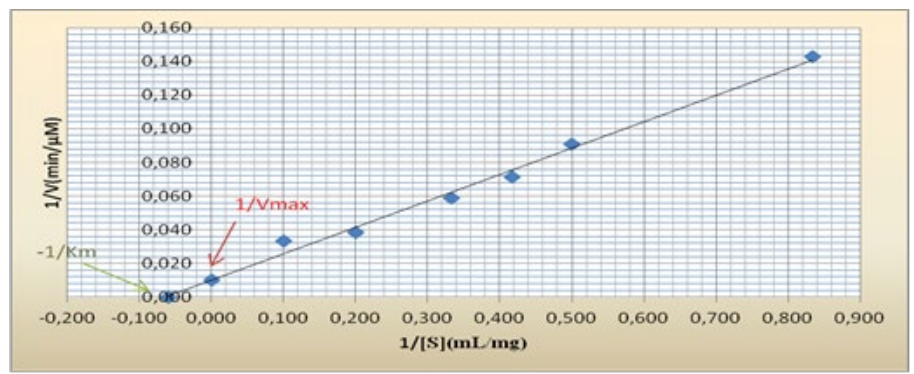

Figura 5: Representación de Lineweaber-Burk: Inversos de las velocidades enzimáticas de la $\alpha$-amilasa de Chenopodium quinoa. Var. Hualhuas blanca frente a los inversos de las concentraciones de sustrato.

Efecto de $\mathrm{pH}$ y temperatura sobre la velocidad de reacción.

En la figura 6 se aprecia que la máxima actividad enzimática de la $\alpha$-amilasa de Chenopodium quinoa var. hualhuas blanca se obtuvo a $\mathrm{pH}$ neutro $(\mathrm{pH}: 7)$ cuya velocidad de reacción es $20 \mu \mathrm{M} / \mathrm{min}$ y la mínima velocidad se obtuvo a $\mathrm{pH}$ ácido cuya velocidad registrada es $4 \mu \mathrm{M} / \mathrm{min}$. Dicha enzima presenta actividad máxima en el rango de $\mathrm{pH}$ : 5.6-7. Este dato concuerda con lo obtenido por Saleh, quien reportó que el $\mathrm{pH}$ óptimo de la a-amilasa de Triticum aestivum (trigo) se encuentra en el rango de 5.0 a 7.0. (Saleh et al., 2009). Sin embargo, se observa que la actividad enzimática es muy baja a $\mathrm{pH}$ ácido: $\mathrm{pH}$ 3.8. El pH óptimo para las $\alpha-$ amilasas de cereales y microorganismos es variable, pero se encuentra generalmente en el rango 6-0-7.0. Existen pocos reportes de esta enzima con un $\mathrm{pH}$ óptimo por debajo de 5.0. Algunos casos excepcionales incluyen la alfa amilasa producida por Alicyclobacillus acidocaldarius con un pH óptimo de 3.0 y la producida por Pyrococcus furiosus activa a un $\mathrm{pH}$ de 3.5. (Quintero et al., 2009). 


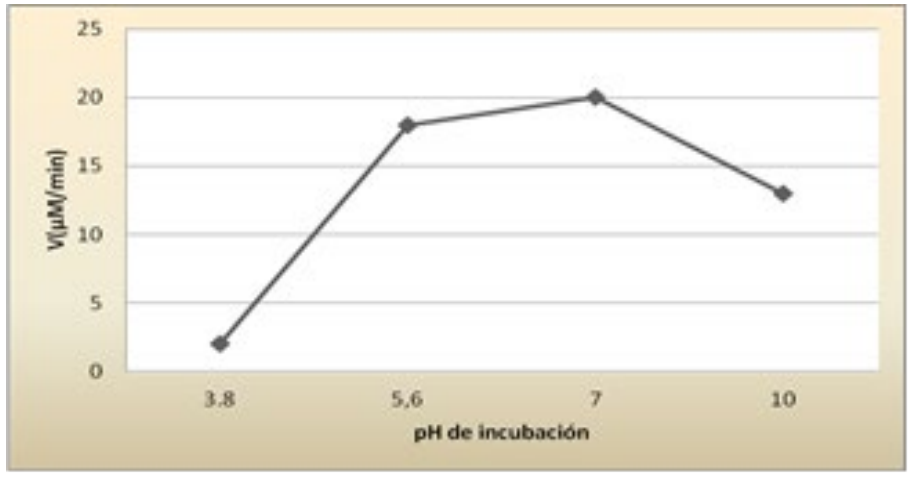

Figura 6. Efecto del pH sobre la velocidad de reacción de $\alpha$ - amilasa de Chenopodium quinoa var. hualhuas blanca.

En la figura 7, se aprecia que la máxima actividad enzimática se obtuvo a $37^{\circ} \mathrm{C}$ cuya velocidad de reacción es $17 \mu \mathrm{M} / \mathrm{min}$. Además, se puede apreciar que sigue manteniendo estabilidad a $4^{\circ} \mathrm{C}$ y $100^{\circ} \mathrm{C}(11$ y $7 \mu \mathrm{M} / \mathrm{min}$ respectivamente). La temperatura óptima de la a-amilasa de Chennopodium quinoa. var. hualhuas blanca $\left(37^{\circ} \mathrm{C}\right)$ es la misma de Oriza sativa (arroz) y Pachyrhizus erosus (yacón) que fueron reportados por Abe y Noman (Maher et al., 2011). Sin embargo, las $\alpha$-amilasas de otros cereales como de Triticum sp. (trigo) presentan mayor estabilidad y el rango de la temperatura óptima es de $40-50^{\circ} \mathrm{C}$. Comparando con la de microorganismos, la mayoría de a-amilasas producidas por bacterias del género Bacillus presentan una temperatura óptima cercana a los 70 y $100^{\circ} \mathrm{C}$ (Quintero, et al. 2009).

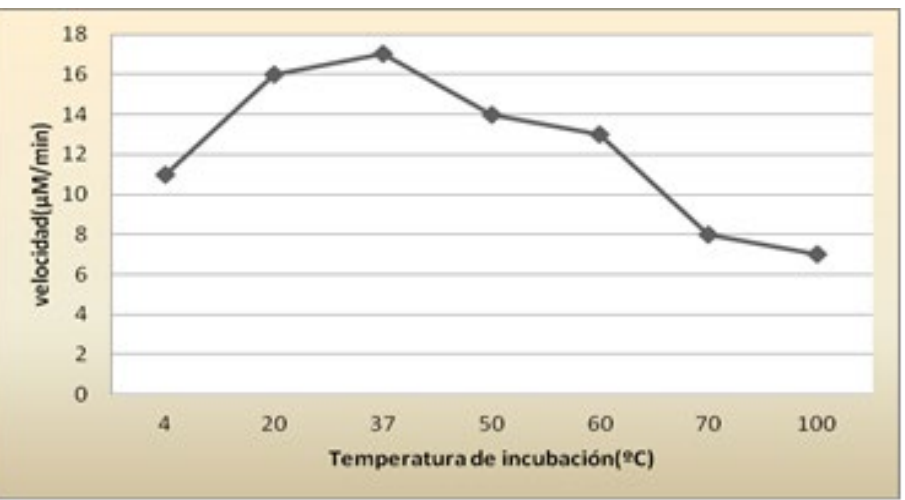

Figura 7: Efecto de la temperatura sobre la velocidad de reacción de a-amilasa de Chenopodium quinoa var. hualhuas blanca.

\section{Conclusiones}

El tiempo de germinación de las semillas de Chenopodium quinoa (quinua) var. hualhuas blanca donde la $\alpha$ - amilasa tiene mayor actividad enzimática es de 36 horas y presenta un peso molecular estimado de $44 \mathrm{kDa}$.
La enzima a-amilasa de Chenopodium quinoa (quinua) var. hualhuas blanca presenta una baja afinidad por el almidón soluble $(\mathrm{Km}: 16 \mathrm{mg} / \mathrm{mL})$ y llega a su máxima actividad a $37^{\circ} \mathrm{C}$ y $\mathrm{pH}: 7$ que es similar a la de los cereales.

\section{Agradecimientos}

Un agradecimiento especial a los integrantes del laboratorio de Bioquímica y Biología molecular de la Universidad Federico Villarreal por su apoyo en el desarrollo del proyecto y a la institución por brindarnos sus instalaciones.

\section{Referencias}

[1] A. Kumari, VK Singh, J. Fitter, T. Polen, AM Kayastha. a-Amylase from germinating soybean (Glycine max) seeds - Purification, characterization and sequential similarity of conserved and catalytic amino acid residues. Phytochemistry, 71 (2010) 1657-1666.

[2] A. Wisessing, A. Engkagul, A. Wongpiyasatid y K. Choowongkomon. Biochemical characterization of the alpha-amylase inhibitor in mungbeans and its application in inhibiting the growth of Callosobruchus maculatus. J. Agric. Food Chem. 58 (4) (2010) 2131-7.

[1] R. Repo-Carrasco, C. Espinoza y S. Jacobsen. Nutritional value and use of the Andean crops quinoa (Chenopodium quinoa) and kan iwa (Chenopodium pallidicaule). Food Rev. Int. 19 (2003) 179 - 189.

[2] L. Abugoch, N. Romero, C. Tapia, J. Silva y M. Rivera. Study of some physico-chemical and functional properties of quinoa (Chenopodium quinoaWilld.) protein iso-lates. J. Agric. Food Chem. 56 (2008) 4745 - 4750.

[3] J. Borges, R. C. Bonomo, C. D. Paula, C. L. Oliveira, y C. M. Cesário. Características Físico-Químicas, Nutricionais e formas de consumo da quinoa (Chenopodium quinoa Willd). TEMAS AGRARIOS 15: (1) 2010) 9 23.

[6] A. Ismail, E. S. Ella, G. V. Vergara y D. J. Mackill. Mechanisms associated with tolerance to flooding during germination and early seedling growth in rice (Oryza sativa). Ann Bot. 0 3(2) (2009) 197-209.

[7] O. Adewale y O. Okoronkwo. Comparative studies on a-amylases from malted maize (zea mays), finger millet (eleusine coracana) and sorghum (sorghum bicolor). Carbohyd polym. 77 (2006) 71-74.

[8] E. Espinel y E. Lopez. Purificación y 
caracterización de a-amilasa de penicillium commune producida mediante fermentación en fase sólida. Revista Colombiana de Química. 38 (2009)

[9] J. Machaiah y U. Vakil. Isolation and partial characterization of $\alpha$-amylase components evolved during early wheat germination. (Bhabha Atomic Research Center, 1984) Vol 6.

[10] A. Maher, A. Maqtari, M. Khalid y S. Hazmy. Identification and characterization of $\alpha$ amylase from yemeni bean (dolichos lablab) seeds. Jordan Journal of Chemistry. 6 (2) (2010)

[11] M. Quintero, O. Montoya y P. Gutierrez. Purificación y Caracterización de una aamilasa producida por la cepa nativa bacillus sp.bbm1. Grupo de biotecnología microbiana. (Universidad Nacional de Colombia Sede Medellín, 2009)

[12] A. Saleh, M. Abdulrahman y L. Malki. Partial purification and characterization of five $\alpha$ amilases from a wheat local variety during germination. Australian Journal of Basic \& Applied Sciences. 3 (2009) 1740

D. Warner, M. Grove y C. Knutson. Isolation and characterization of $\alpha$-amylases from endosperm of germinating maize. Cereal Chern. 68(4) (1991) 383-390

[14] P. Monteiro de Souza y P. de Oliveira. Application of Microbial $\alpha$-Amilase in industry.Brasilian journal of microbiology. (2010) 850-861.

E-mail: melistart239@hotmail.com oscarnol@gmail.com anaisabelflor@gmail.com psantacruzcm@yahoo.es 